\title{
A Fundação Oswaldo Cruz e a ciência no feminino: a participação feminina na prática $e$ na gestão da pesquisa em uma instituição de ensino e pesquisa*
}

\author{
Jeorgina Gentil Rodrigues** \\ Maria Cristina Soares Guimarães***
}

\section{Resumo}

Este estudo teve por objetivo delinear a participação feminina no esforço de pesquisa realizado pela Fundação Oswaldo Cruz (Fiocruz), especialmente nos anos recentes. A fonte inicial para levantamento de dados foi a Diretoria de Recursos Humanos (Direh) da Fiocruz, com vistas a identificar o contingente de servidores e servidoras com titulação de doutorado. Consulta ao Portal Transparência permitiu identificar o total de servidore/as que entraram por concurso público, e que no momento do estudo possuíam titulação de doutorado. $\mathrm{O}$ passo seguinte foi a busca dos respectivos currículos cadastrados na Plataforma Lattes, utilizando-se a ferramenta ScriptLattes. As análises realizadas cobriram o período 1996-2013. Os resultados apontam que ainda que a produção bibliográfica das mulheres em números absolutos seja maior que a dos homens, eles apresentam produção média superior a das mulheres. Em paralelo, uma segunda perspectiva de análise documental foi realizada com vistas a mapear a participação feminina em postos de tomada de decisão na Fiocruz. Os dados sugerem, no geral, segregação hierárquica (ou vertical), fenômeno conhecido na literatura como "teto de vidro", que caracteriza-se pela menor velocidade das mulheres na ascensão da carreira, em comparação com a progressão profissional masculina, o que resulta na sub-representação das mulheres nos postos de tomada de decisão e, consequentemente, limita o alcance de posições de maior prestígio na instituição.

Palavras-chave: Gênero e Ciência, Produção Científica Feminina, Fundação Oswaldo Cruz.

* Recebido para publicação em 11 de junho de 2015, aceito em 16 de novembro de 2015.

** Doutora em Informação, Comunicação em Saúde do Icict/Fiocruz - Biblioteca de Manguinhos, Icict/Fiocruz. Rio de Janeiro, RJ, Brasil. jeorgina.gentil@icict.fiocruz.br

**: Doutora em Ciência da Informação, UFRJ/IBICT - Laboratório de Informação Científica e Tecnológica em Saúde, Icict/Fiocruz. Rio de Janeiro, RJ, Brasil. cristina.guimaraes@icict.fiocruz.br 
The Oswaldo Cruz Foundation and Science on Women: Women's Participation in Practice and Research Management in an Educational and Research Institution

\begin{abstract}
This study aimed to delineate women's participation in recent research conducted by the Oswaldo Cruz Foundation (Fundação Oswaldo Cruz - Fiocruz). The initial data source was the Human Resources Directory (Diretoria de Recursos Humanos - Direh) at Fiocruz, which was used to identify the number of employees with doctorates. The ScriptLattes tool was then used to search for curriculum vitae $(\mathrm{CV})$ that were registered in the Lattes Platform for the period from 1996 to 2013. The results indicate that although women's bibliographic production in absolute numbers is greater than men's, they have an average production performance higher than women's. A second document analysis was performed to map women's participation in decision-making positions at Fiocruz. In general, the data suggest hierarchical (or vertical) segregation, a phenomenon known in the literature as the "glass ceiling", which is characterized by a slower rise in career for women compared to men. This disadvantage results in the underrepresentation of women in positions of decision-making, and women are limited from reaching the most prestigious positions in the institution.
\end{abstract}

Key Words: Gender and Science, Female Scientific Production, Oswaldo Cruz Foundation. 


\section{Introdução}

Em anos recentes, e quase meio século após o movimento feminista ganhar seus primeiros contornos em diferentes campos discursivos em perspectiva internacional, as assimetrias de gênero ${ }^{1}$ passaram a fazer parte integral da agenda política de diversas sociedades (ainda que permaneçam ausentes das agendas políticas de diversos países da África subsaariana e da maioria das sociedades muçulmanas). No Brasil, talvez por força do compromisso assumido com as Metas do Milênio ${ }^{2}$ ou, quiçá, em decorrência de um crescimento expressivo da participação das mulheres em posição de destaque na sociedade, as ações $e$ políticas referentes às mulheres foram integradas no âmbito do Plano Nacional de Políticas para as Mulheres (Brasil, 2005) e contam com uma Secretaria de Políticas para as Mulheres (SPM), com status de ministério, vinculada à Presidência da República. A Lei $\mathrm{n}^{\circ}$ 11.340, conhecida como Lei Maria da Penha, que entrou em vigor em 22 de setembro de 2006, é um marco no cumprimento de garantias constitucionais sobre o direito das mulheres a uma vida livre de violência.

A partir de 2011, e já com uma mulher presidindo o país, as organizações públicas e privadas brasileiras podem, voluntariamente, aderir ao Programa Pró-Equidade de Gênero e Raça e se candidatarem a ganhar o Selo Pró-Equidade de Gênero e Raça pelo compromisso com a eliminação de todas as formas de discriminação no acesso, remuneração, ascensão e permanência no emprego (Brasil, 2011). Um dos focos do programa está no quesito "cultura organizacional", o que de longe (e de muitos) se

\footnotetext{
1 Conforme Scott (1990:14), gênero é "[...] um elemento constitutivo de relações sociais fundadas sobre as diferenças percebidas entre os sexos, e o gênero é um primeiro modo de dar significado às relações de poder".

2 Na reunião de Cúpula do Milênio, realizada em Nova Iorque, em 2000, a "promoção da igualdade entre os gêneros e o empoderamento das mulheres" ganhou destaque como a terceira das oito Metas do Milênio pactuadas pela Assembleia das Nações Unidas, com relação ao desenvolvimento global até 2015.
} 
sabe ser o cenário que, se não se justifica, é invariavelmente mobilizado para explicitar as assimetrias de poder entre homens e mulheres. ${ }^{3}$ Esse é o caso, por excelência, de grande parte das discussões de gênero no domínio da ciência, ou seja, "consiste em fazer ciência mantendo os valores dominantes da cultura científica tradicional" (Oliveira; Amâncio, 2006:598), ou, de uma ciência que é, essencialmente, masculina.

O modelo e a cultura masculinos de sucesso na ciência (Oliveira; Amâncio, 2006) envolvem relações acadêmicas competitivas entre pares e o compromisso integral com o trabalho científico. A trajetória e as estratégias que possibilitam que as mulheres "cheguem lá" implicam enfrentar e superar dificuldades e barreiras de grande monta, barreiras essas que começaram a ser construídas nos processos de socialização diferenciados de meninas e meninos (Velho, 2006).

De fato, a sub-representação das mulheres em certos ramos da ciência tem gerado um debate considerável. Em 1971, o estudo de Lewin e Duchan assinalou a pouca motivação das mulheres quanto ao seu desempenho acadêmico devido à discriminação considerada como uma forma de "pressão invisível" (unseen pressure) - com respeito aos critérios de promoção (Lewin; Duchan, 1971:892). Trinta e quatro anos depois, Lawrence H. Summers, então reitor da Universidade de Harvard, nos Estados Unidos, ficou conhecido por afirmar que as causas para haver menos mulheres na área de Ciências Exatas estão ligadas às diferenças cerebrais entre os gêneros. A declaração provocou um frisson internacional e ilustra a natureza profundamente enraizada dos preconceitos nesse debate (Agrello; Garg, 2009; Summers, 2005).

Não é de se estranhar, portanto, que as instituições de Ciência e Tecnologia (C\&T), em alguns casos, se esqueçam de considerar as lutas e conquistas obtidas pelo movimento feminista

3 Apesar dos progressos verificados em relação à maior participação das mulheres no mercado de trabalho, registram-se ainda assimetrias de poder, remuneração e prestígio entre homens e mulheres, mesmo no caso em que um dado homem e uma dada mulher, ambos hipotéticos, possuem qualificações profissionais idênticas (Rodrigues, 2014). 
e não repensem as relações de gênero presentes nas instituições (Osada; Costa, 2006). Os espaços conquistados pelas mulheres na ciência são o resultado de esforço pessoal. Embora as mulheres estejam atuando de forma mais intensa em instituições de pesquisa e ensino, ainda enfrentam a discriminação $e$ a desigualdade de gênero, expressas nas mais diferentes perspectivas (Leta, 2003).

A despeito de inúmeras estratégias de mobilização $e$ inserção progressiva das mulheres em $\mathrm{C} \& \mathrm{~T}$ em todo o mundo (Unesco, 2005), são ainda escassos os dados sistematizados, em âmbitos nacional e internacional, sobre a formação, trajetória $e$ perfil dos recursos humanos na ciência, com foco em características específicas, como gênero, estrato social ou raça/etnicidade (Leta, 2003).

As possíveis expressões de iniquidades/segregação de gênero na carreira científica seriam, portanto, discerníveis tão somente a partir de análises de dados desagregados que levem em conta as eventuais especificidades por área do conhecimento, juntamente com a assim denominada segregação hierárquica (ou vertical). Esta última é conhecida na literatura internacional como "teto de vidro" (glass ceiling), e caracteriza-se pela menor velocidade na ascensão da carreira pelas mulheres, em comparação com a progressão profissional masculina, o que resulta na sub-representação das mulheres nos postos de tomada de decisão e, consequentemente, limita o alcance de posições de maior prestígio e o acesso a uma melhor remuneração (Citeli, 2000; Costa, 2006; Lima, 2013; Olinto, 2011; Schiebinger, 2001; Vaz, 2010, 2013).

Apesar de alguns progressos verificados em relação à igualdade de participação de mulheres e homens no mercado de trabalho, a situação das mulheres no domínio das áreas de Ciência, Tecnologia, Engenharia e Matemática (CTEM) continua deficitária (Etzkowit; Kemelgor e Uzzi, 2003).

Estudiosos/as sobre o assunto (Etzkowitz, Kemelgor, Uzzi, 2003; Lane, Goh, Driver-Linn, 2012; Saavedra, Taveira, Silva, 2010; Xu, 2008) apontam que nessas áreas segue-se um escrutínio sobre os 
estereótipos de gênero que ainda persistem em diversas sociedades devido a uma "cultura científica" centrada em valores masculinos.

Para mitigar a desigualdade, ou buscar maiores equilibrio $e$ proporcionalidade entre gêneros em C\&T, é necessário estimular a inserção e a participação das mulheres, e para isso são propostas algumas ações: 1) promover a igualdade de oportunidades das mulheres no acesso aos programas de pesquisa, desenvolvimento $e$ inovação; 2) oferecer oportunidades de networking ${ }^{4}$ e de capital social; 3) garantir estrutura e flexibilidade de e no local de trabalho; 4) estimular apoio e financiamento de projetos de pesquisa e financiamentos; 5) conceder licença gestante para bolsistas nas diferentes fases de formação científica; e 6) garantir creches e ações relacionadas. Em países campeões no quesito "igualdade de gênero", como a Suécia e a Dinamarca, congressos científicos, como na área de física, são equipados com espaços para os filhos dos cientistas - sejam eles mulheres ou homens (Araújo-Jorge et al., 2013).

Entre as ações voltadas à inserção feminina na ciência, desde 1993, a Organization for Women in Science for the Developing World (OWSD) oferece bolsas de estudo a mulheres em formação científica com o objetivo de reforçar o seu papel e sua inserção no desenvolvimento em seus respectivos países, aumentar sua produtividade científica e formar líderes que atuem em processos decisórios nos níveis nacional e internacional (Araújo-Jorge et al., 2013).

De outra frente, mais especificamente, o relatório Mapping the maze: getting more women to the top in research (European Commission, 2008) revela que, embora processos de avaliação $e$ promoção justos e transparentes sejam instrumentos necessários à

4 Networking (em inglês) é uma expressão que representa uma rede de contatos. Diz respeito às pessoas que um indivíduo conhece e aos relacionamentos pessoais, comerciais e profissionais que mantém com elas. Disponível em: <http://pt.wikipedia.org/wiki/Networking>. Acesso em: 20 jan. 2012. 
equidade de gênero ${ }^{5}$, por si sós, não são suficientes: é indispensável que ocorra uma mudança de cultura para alcançar uma representação mais equilibrada de homens e de mulheres nos órgãos de decisão.

Há aqueles que advogam, também, que a análise dos processos de produção, difusão e uso do conhecimento científico, à luz do crescimento exponencial da ciência a partir de meados do século $\mathrm{XX}$, não tem sido sensível à temática de gênero, ignorando que mulheres $e$ homens têm trajetórias diferenciadas, e que desvelar as mulheres no mundo científico e tecnológico exige um esforço específico.

A menor produtividade das mulheres é ressaltada em inúmeros estudos referentes aos mais variados campos científicos, observados durante longos períodos e em diferentes países (Ferreira et al., 2008:46). Segundo os autores, nesses estudos, verifica-se que ao longo da carreira, na média, mulheres publicam menos da metade do que os pares homens, o que sugere que o sexo constitui uma importante fonte de variação e se correlaciona à publicação. Rossiter (1993:327) observa também que as diferenças entre os sexos tendem a acumular desvantagens para as mulheres e vantagens para os homens, e são determinantes para o desenvolvimento da carreira ainda durante a formação em nível de doutoramento. Ferreira et al. (2008:46) concluem que não há consenso em relação a essas disparidades, nem existem indicadores universalmente aceitos para mensurar de forma adequada os diferentes níveis de produtividade entre os homens $e$ mulheres.

Por outro lado, as pesquisas de Xie e Shauman (2003) mostram que o estatuto familiar (casamento, presença de filhos) não tem influência significativa na produtividade, quando são controladas as principais variáveis explicativas, a saber,

${ }^{5}$ Equidade de gênero se refere ao estágio de desenvolvimento humano no qual direitos, responsabilidades e oportunidades de indivíduos não serão determinados pelo fato de que indivíduos de diferentes sociedades tenham nascido homem ou mulher (Fórum Econômico Mundial, 2005:2). 
diminuição de produtividade entre os sexos com o passar dos anos, efeito positivo da qualidade da instituição na produtividade, efeito negativo entre a obtenção do bacharelado e doutorado na produtividade do pesquisador, e o tipo de instituição a que pertence (grau de prestígio, parte correspondente a horas de ensino e de pesquisa).

Produtividade, carreiras típicas, casamento, maternidade, etnia, padrão social e cultural, mobilidade, perfil de cooperação, mentoring ${ }^{6}$ são algumas das inúmeras dimensões que têm guiado as pesquisas e alimentado a aparente infindável discussão sobre os desempenhos masculino e feminino na ciência, que tem gerado um número limitado de orientações para as políticas públicas.

Segundo Smith-Doerr (2004), a carreira científica feminina parece ganhar dinâmicas diferentes quando fora do ambiente profundamente hierárquico da academia. A autora sugere que um conjunto de fatores, como rigidez organizacional e profissional $e$ atitude social, dificulta a carreira feminina em maior grau na academia (leia-se universidades) do que em laboratórios $e$ institutos de pesquisa. Em outras palavras, em instituições que estimulem o trabalho em rede, a parceria $e$ as habilidades também são mais altamente recompensadas.

No Brasil, as pesquisas no campo de ciência e gênero começaram a ganhar corpo a partir do início dos anos 2000 (Leta, 2003; Leta, Lewison, 2003; Lopes, 2002; Osada, Costa, 2006; Tabak, 2002; Velho, Prochazka, 2003). Os estudos nacionais tomam como fonte de dados o agregado quantitativo da produção científica nacional (Leta, 2003), e ainda pouca luz tem sido lançada sobre a participação feminina em disciplinas científicas particulares. Estudiosos têm apontado a importância de estudos de nível micro, tanto disciplinar quanto institucional, que possam explicar a concentração de mulheres em determinadas carreiras científicas em detrimento de outras (Mason, Goulden, 2003). Esse fenômeno, conhecido como gender tracking, "tem se mostrado extremamente

\footnotetext{
${ }^{6}$ Mentoring é uma relação de trabalho entre um membro mais experiente e um iniciante com uma agenda designada à troca de experiência e aprendizado.
} 
persistente e refratário a mudanças, mesmo na presença de políticas destinadas a enfrentá-lo" (Moreira \& Velho, 2010:282).

É por meio de estudos em perspectiva micro que pesquisadores têm questionado a desigualdade de gênero na ciência, a partir de explicações que postulam que formas sutis de discriminação persistem no local de trabalho e limitam o avanço das mulheres na carreira científica. Tais análises apontam para crenças $e$ atitudes que operam inconscientes por meio de interações de trabalho e de políticas e procedimentos subjetivos, institucionalizados no local de trabalho (Roos \& Gatta, 2007).

Orientada pela importância dessas análises em perspectiva micro, o objetivo desta pesquisa foi delinear os contornos da participação feminina na prática e na gestão da pesquisa em ciências de saúde no Brasil, a partir de uma instituição de ensino e pesquisa, a Fundação Oswaldo Cruz (Fiocruz), considerada uma das principais instituições públicas de C\&T no campo da saúde do país (Mello \& Amâncio Filho, 2010). Tomando como ponto de partida a meritocracia (concurso público) no acesso à instituição $e$ à equidade como princípio de progressão na carreira, cabe perguntar como vem se desenhando a participação feminina na pesquisa na Fiocruz (Rodrigues, 2014).

\section{Fiocruz e a ciência no feminino}

O período de cobertura desta presente pesquisa vai de 1996 até 2013. Foi realizada uma consulta à Diretoria de Recursos Humanos (Direh) com vistas a identificar a força de trabalho da Fiocruz. Na distribuição da força de trabalho da fundação, há um leve predomínio do sexo feminino, sendo que os homens representam $49,19 \%$ (6.175) e as mulheres $50,81 \%$ (6.378). Na modalidade "servidores públicos", observa-se que a Fiocruz tem um número maior de servidoras (2.969), 32,49\% do que de servidores (2.241). No caso dos "terceirizados", ocorre o inverso, o maior número é de trabalhadores (3.910), 15,95\% superior ao de trabalhadoras (3.372). Identificadas as modalidades da força de 
trabalho na Fiocruz, a modalidade "servidores públicos" foi considerada para este estudo (Fundação Oswaldo Cruz, 2013b).

A Fiocruz, entre 1996 e 2010, realizou cinco concursos públicos e incorporou 3.128 novos servidores, o que representa $60 \%$ dos servidores ativos na atualidade (Fundação Oswaldo Cruz, 2013b), sendo 1.233 tecnologistas, 699 analistas, 591 técnicos, 488 pesquisadores, 99 assistentes e 18 especialistas.

Com o objetivo de identificar os servidores e servidoras, com titulação de doutorado ${ }^{7}$, no momento do estudo, independente de onde estivessem locados na fundação, se em ensino e pesquisa ou em outras modalidades de gestão, uma segunda consulta à Direh foi realizada. Assim, do total de 5.210 servidores, até maio de 2012, foram identificados 1.064 (22.43\%) servidores com titulação de doutorado, sendo 654 (61,5\%) mulheres e 410 (38,5\%) homens, o que evidencia uma qualificação compatível com uma instituição de pesquisa (Quadro 1).

Dado o quantitativo de servidores com titulação de doutorado e que tiveram acesso à instituição por diferentes mecanismos, optou-se por trabalhar apenas com as admissões por concursos públicos realizadas após a promulgação da Constituição federal de 1988.

7 Concessão de retribuição de titulação e gratificação por qualificação, no caso doutorado, pela Direh segundo a Lei $n^{\circ}$ 11.355/2006 e o Decreto $n^{\circ} 7.922 / 2013$ (Fundação Oswaldo Cruz, 2013b). 
cadernos pagu (46) Jeorgina G. Rodrigues e Maria Cristina S. Guimarães 207

Quadro 1 - Total de servidores com titulação de doutorado (maio de 2012)

\begin{tabular}{|l|c|c|c|}
\hline \multicolumn{1}{|c|}{ INSTÂNCIA } & F & M & TOTAL GERAL \\
\hline BIOMANGUINHOS & 12 & 9 & 21 \\
\hline COC & 25 & 18 & 43 \\
\hline CPqAM & 39 & 26 & 65 \\
\hline CPqGM & 16 & 21 & 37 \\
\hline CPqLMD & 6 & 7 & 13 \\
\hline CPqRR & 34 & 22 & 56 \\
\hline CRPHF & 1 & 0 & 1 \\
\hline ENSP & 113 & 77 & 190 \\
\hline EPSJV & 19 & 12 & 31 \\
\hline FARMANGUINHOS & 18 & 8 & 26 \\
\hline ICC & 1 & 3 & 4 \\
\hline ICICT & 11 & 11 & 22 \\
\hline IFF & 62 & 28 & 90 \\
\hline INCQS & 27 & 8 & 35 \\
\hline IOC & 180 & 110 & 290 \\
\hline IPEC & 55 & 31 & 86 \\
\hline PRESIDÊNCIA & 35 & 19 & 54 \\
\hline TOTAL GERAL & $\mathbf{6 5 4}$ & $\mathbf{4 1 0}$ & $\mathbf{1 . 0 6 4}$ \\
\hline
\end{tabular}

Fonte: Dados disponibilizados pela Direh/Fiocruz (2012).

Para identificar os/as servidores/as concursados/as foi a realizado um levantamento de dados no Portal Transparência (http://www.portaltransparencia.gov.br/), com objetivo de identificar o ato de nomeação da servidora ou servidor, por portaria e consulta. Dessa seleção, do total de 1.064 doutores, 571 $(53,7 \%)$ ingressaram por meio de concursos públicos realizados a partir de 1996. Desses, 346 (60,6\%) mulheres e 225 (39,4\%) homens com título de doutor foram admitidos por concurso entre 1996 e 2010, como apresentado no Quadro 2, a seguir: 
Quadro 2 - No de servidores com título de doutor e admitidos por concurso (de 1996 a 2010)

\begin{tabular}{|l|c|c|c|}
\hline \multirow{2}{*}{\multicolumn{1}{|c|}{ UNIDADE }} & \multicolumn{2}{c|}{ SEXO } & \multirow{2}{*}{ TOTAL } \\
\cline { 2 - 3 } & $\mathbf{F}$ & $\mathbf{M}$ & GERAL \\
\hline BIOMANGUINHOS & 9 & 3 & 12 \\
\hline COC & 16 & 7 & 23 \\
\hline CPqAM & 24 & 17 & 41 \\
\hline CPqGM & 14 & 9 & 23 \\
\hline CPqLMD & 5 & 7 & 12 \\
\hline CPqRR & 27 & 17 & 44 \\
\hline ENSP & 33 & 28 & 61 \\
\hline EPSJV & 14 & 7 & 21 \\
\hline FARMANGUINHOS & 12 & 7 & 19 \\
\hline ICICT & 9 & 6 & 15 \\
\hline IFF & 22 & 14 & 36 \\
\hline INCQS & 14 & 3 & 17 \\
\hline IOC & 91 & 65 & 156 \\
\hline IPEC & 34 & 20 & 54 \\
\hline PRESIDENCIA & 22 & 15 & 37 \\
\hline TOTAL GERAL & $\mathbf{3 4 6}$ & $\mathbf{2 2 5}$ & $\mathbf{5 7 1}$ \\
\hline
\end{tabular}

Fonte: Dados coletados no Portal Transparência (2013).

A próxima etapa foi a busca dos respectivos currículos dos/as servidores/as com titulação de doutorado cadastrados/as na Plataforma Lattes (http://lattes.cnpq.br/) para identificar os registros relativos à vida acadêmica de cada um/a deles/as.

Esse conjunto de currículos foi baixado em uma base de dados especialmente desenhada para recebê-los e, em seguida, o total de referências foi migrado e tratado em um software de mineração de dados, o VantagePoint ${ }^{\circledR}$, o que permitiu análises quantitativas da produção acadêmica e técnica, das orientações, do acesso às bolsas de produtividade e de prêmios.

Apenas um currículo não pode ser encontrado na Plataforma Lattes referente a uma servidora do Instituto de Pesquisa Clínica Evandro Chagas (Ipec), atual Instituto Nacional de Infectologia Evandro Chagas (INI), o que delimita o corpus de análise em 570 currículos cadastrados na Plataforma Lattes, sendo 345 currículos de doutoras e 225 currículos de doutores. 
Produção bibliográfica global de mulheres e homens

Ao longo do período de 1996-2013, o Lattes registra um total de 30.757 itens classificados em "produção bibliográfica" (artigos publicados, livros publicados, capítulos de livro, textos em jornais e revistas, trabalhos completos em anais, resumos expandidos em anais, resumos em anais, artigos aceitos, apresentações de trabalhos, demais produções), sendo 17.282 de produção bibliográfica de mulheres e 13.475 de produção bibliográfica de homens, como sintetizado no Quadro 3.

O artigo científico foi a produção científica mais valorizada no período analisado. Ainda que a produção das mulheres em números absolutos seja maior que a dos homens, a média de artigos publicados pelos homens (19,2 artigos/homem) é 51,8\% maior que a produção pelas mulheres (12,6 artigos/mulher), segundo o comparativo da produção global.

Também é possível verificar a diferença percentual entre as médias de produção dos homens e das mulheres. Com mais de $50 \%$ de artigos completos publicados e capítulos de livros, os homens também têm $26,6 \%$ mais livros publicados, $30 \%$ mais trabalhos completos em anais de congressos, com uma produção bibliográfica geral $19,6 \%$ maior. Em relação à missão de transferência de conhecimento para a sociedade utilizando outros instrumentos além da publicação científica, as mulheres produziram $24 \%$ a mais que os homens na modalidade "textos em jornais e revistas". 


\section{Quadro 3 - Produção de homens e mulheres concursados da Fiocruz e com doutorado}

\begin{tabular}{|c|c|c|c|c|c|}
\hline \multirow{2}{*}{ TIPO DE PRODUÇÄO } & \multicolumn{2}{|c|}{ HOMENS } & \multicolumn{2}{|c|}{ MULHERES } & \multirow{2}{*}{$\begin{array}{l}\text { DIFERENÇA \% } \\
\text { ENTRE AS MÉDIAS } \\
\text { HOMEM-MULHER }\end{array}$} \\
\hline & QTDE & MÉDIA & QTDE & MÉDIA & \\
\hline \multicolumn{6}{|l|}{ PRODUÇÃO BIBLIOGRÁFICA } \\
\hline Artigos Completos & 4.321 & 19,2 & 4.364 & 12,6 & $51,8 \%$ \\
\hline Livros Publicados & 142 & 0,6 & 172 & 0,5 & $26,6 \%$ \\
\hline Capitulos de Livros & 695 & 3,1 & 710 & 2,1 & $50,1 \%$ \\
\hline Textos em Jornais e Revistas & 276 & 1,2 & 557 & 1,6 & $-24,0 \%$ \\
\hline Trabalhos Completos em Anais & 737 & 3,3 & 869 & 2,5 & $30,0 \%$ \\
\hline Resumos Expandidos em Anais & 228 & 1,0 & 334 & 1,0 & $4,7 \%$ \\
\hline Resumos em Anais & 4.837 & 21,5 & 7.122 & 20,6 & $3,9 \%$ \\
\hline Apresentaçōes de Trabalho & 1.943 & 8,6 & 2.770 & 8,0 & $7,6 \%$ \\
\hline Demais Produçōes & 251 & 1,1 & 338 & 1,0 & $13,9 \%$ \\
\hline Total de Produção Bibliográfica & 13.475 & 59,9 & 17.282 & 50,1 & $19,6 \%$ \\
\hline \multicolumn{6}{|l|}{ PRODUÇÃO TÉCNICA } \\
\hline Produtos Tecnológicos & 57 & 0,3 & 115 & 0,3 & $-24,0 \%$ \\
\hline Processos ou Técnicas & 93 & 0,4 & 66 & 0,2 & $116,1 \%$ \\
\hline Trabalhos Técnicos & 750 & 3,3 & 1.562 & 4,5 & $-26,4 \%$ \\
\hline Demais Tipos de Produção Técnica & 997 & 4,4 & 1.430 & 4,1 & $6,9 \%$ \\
\hline Total de Produção Técnica & 1.897 & 8,4 & 3.173 & 9,2 & $-8,3 \%$ \\
\hline \multicolumn{6}{|l|}{ PRODUÇÃO ARTÍSTICA } \\
\hline Total de Produção Artistica & 60 & 0,3 & 21 & 0,1 & $338,1 \%$ \\
\hline \multicolumn{6}{|l|}{ ORIENTAÇÕES CONCLUÍDAS } \\
\hline Pós-Doutorado & 121 & 0,5 & 86 & 0,2 & $115,7 \%$ \\
\hline Doutorado & 422 & 1,9 & 379 & 1,1 & $70,7 \%$ \\
\hline Mestrado & 875 & 3,9 & 1.105 & 3,2 & $21,4 \%$ \\
\hline Conclusão Curso Lato Sensu & 348 & 1,5 & 581 & 1,7 & $-8,2 \%$ \\
\hline Conclusão de Curso de Graduaçăo & 410 & 1,8 & 478 & 1,4 & $31,5 \%$ \\
\hline Iniciação Científica & 723 & 3,2 & 1.022 & 3,0 & $8,5 \%$ \\
\hline Orientaçōes de Outra Natureza & 357 & 1,6 & 594 & 1,7 & $-7,8 \%$ \\
\hline Total de Orientações Concluidas & 3.256 & 14,5 & 4.245 & 12,3 & $17,6 \%$ \\
\hline \multicolumn{6}{|l|}{ PROJETOS DE PESQUISA } \\
\hline Total de Projetos de Pesquisa & 1.376 & 6,1 & 1.893 & 5,5 & $11,5 \%$ \\
\hline \multicolumn{6}{|l|}{ PRÊMIOS E TITULLOS } \\
\hline Total de Prêmios e Títulos & 460 & 2,0 & 490 & 1,4 & $43,9 \%$ \\
\hline \multicolumn{6}{|l|}{ PARTICIPAÇĀO EM EVENTOS } \\
\hline Total de Participação em Eventos & 3.728 & 16,6 & 5.834 & 16,9 & $-2,0 \%$ \\
\hline \multicolumn{6}{|l|}{ ORGANIZAÇÃO DE EVENTOS } \\
\hline Total de Organização de Eventos & 422 & 1,9 & 695 & 2,0 & $-6,9 \%$ \\
\hline
\end{tabular}

Elaboração própria a partir do Lattes (2013).

Em relação à "produção técnica" no período analisado, os dados registrados no currículo Lattes referem-se a palestras e conferências ministradas, participação em bancas de concursos e elaboração de pareceres para agências de fomento e revistas 
científicas. O Quadro 3 evidencia a liderança masculina em quase todas as produções. No item "processos ou técnicas" da "produção técnica" $(116,1 \%)$ de processos ou técnicas declarados. Outro destaque para os homens é o registro três vezes maior (338\%) de obras artísticas. ${ }^{9}$ No geral, as mulheres produzem menos que os homens, mas na produção técnica nos itens "produtos tecnológicos" 10 (24\%) e "trabalhos técnicos" ${ }^{11}(26,4 \%)$ as mulheres apresentaram uma produção superior aos homens.

Foi possível observar a formação de recursos humanos. Foram realizadas 7.501 orientações e supervisões. Contudo, em orientações, os homens têm maior produção em quase todos os itens com destaque para mais que o dobro de supervisões de pósdoutorado e $70 \%$ a mais de orientação de doutorado em relação às mulheres. As mulheres têm maior produção apenas em orientações Lato Sensu e outras orientações, mesmo assim em pequena proporção, não chegando a $10 \%$.

Para atender a missão de pesquisa da Fiocruz, no período analisado, foram desenvolvidos 3.259 projetos de pesquisa de padrão competitivo internacional. No total de projetos de pesquisa, os homens apresentam uma produção superior $(11,5 \%)$ às mulheres.

\footnotetext{
${ }^{8}$ Compreende processos ou técnicas, produtos tecnológicos, trabalhos técnicos e demais tipos de produção técnica.

9 Concentra toda atividade relacionada à área cultural, apresentações de obras, arranjos ou composições musicais, artes visuais, programas de rádio ou TV etc.

${ }^{10}$ Compreende desenvolvimento de softwares, produtos $e$ processos tecnológicos.

${ }^{11}$ Compreende assessoria, consultoria, parecer, elaboração de projeto, relatório técnico, apresentação de trabalho, cursos de curta duração, desenvolvimento de material didático, editoria, organização de evento, programa de rádio e TV etc.
} 


\section{Distribuição das bolsas de produtividade do CNPq entre homens e mulheres}

A bolsa de produtividade do CNPq é uma bolsa destinada aos pesquisadores que se destaquem, visando a valorização da produção científica. A solicitação é feita pelo próprio pesquisador por meio de formulário de proposta online na página do CNPq. Os pesquisadores são divididos por categorias e níveis, que vão do Nível 2, correspondente aos pesquisadores mais jovens que já apresentam produtividade destacada, até o Nível 1-A, que agrupa os pesquisadores de maior senioridade e liderança científica em cada área.

Entre os servidores/as com titulação de doutorado concursados/as, a Fiocruz conta com 113 bolsistas de produtividade do $\mathrm{CNPq}$ (Tabela 1) - verifica-se que entre as mulheres, $64(18,3 \%)$ recebem de bolsa de produtividade do $\mathrm{CNPq}$, e entre os homens esse percentual é 4\% maior, com 50 (22,3\%) dos homens recebendo bolsas. A bolsa mais frequente entre homens e mulheres é a de Nível 2. No Nível 1, os homens apresentam uma frequência de bolsas quase duas vezes maior que as mulheres. Apesar de a proporção de bolsistas mulheres aumentar, diminui na medida em que cresce o nível hierárquico da bolsa.

\section{Tabela 1 - Distribuição por bolsa de produtividade entre servidores/as concursados/as da Fiocruz}

\begin{tabular}{l|c|c|c|c}
\hline BOLSA DE PRODUTIVIDADE DO CNPq & MULHERES & $\%$ MULHERES & HOMENS & $\%$ HOMENS \\
\hline Nivel 1A & 3 & $4,7 \%$ & 5 & $9,4 \%$ \\
Nivel 1B & 3 & $4,7 \%$ & 6 & $11,3 \%$ \\
Nivel 1C & 2 & $3,1 \%$ & 4 & $7,5 \%$ \\
Nivel 1D & 8 & $12,5 \%$ & 4 & $7,5 \%$ \\
Nivel 2 & 47 & $73,5 \%$ & 31 & $58,4 \%$ \\
Total & 63 & $18,3 \%$ & 50 & $22,3 \%$ \\
\hline
\end{tabular}

Fonte: Elaboração própria a partir do Lattes (2013). 
No período analisado, outros tipos de bolsa foram identificados, como as bolsas para a formação de recursos humanos, em nível de pós-graduação. Foram identificadas 1 bolsa de pós-doutorado sênior, sendo $1(1,6 \%)$ bolsista mulher e 3 bolsas de pós-doutorado no exterior, sendo $3(5,7 \%)$ bolsistas homens.

\section{Cargos comissionados}

Os cargos comissionados (DAS) são postos gerenciais considerados de "confiança", podendo ser ocupados por servidores públicos da própria instituição ou transferidos de outros órgãos, ou mesmo de fora do serviço público. O grau de importância e de poder do cargo está diretamente relacionado com a gradação do DAS. Quanto maior for sua responsabilidade no cargo, maior será o DAS, que varia entre níveis de 1 a 6 . Observa-se que o número de mulheres em postos de tomada de decisão é inversamente proporcional ao nível decisório associado a esses postos, ou seja, quanto mais alto o DAS, menor é a participação feminina (Fontenele-Mourão, 2006).

Em relação à distribuição de cargos comissionados $e$ funções gratificadas na Fiocruz (Decreto $n^{\circ} 4725$ de 9 de junho de 2003, Estatuto da Fiocruz, alterado pelo Decreto $n^{0} 7.171$ de 6 de maio de 2010), a instituição dispõe de 862, entre um e outro, em sua estrutura organizacional (Fundação Oswaldo Cruz, 2013b).

Até o mês de novembro de 2013, 768 cargos estavam ocupados, ou seja, $89,10 \%$ do total, sendo 386 ocupados por servidores e 382 por servidoras (Fundação Oswaldo Cruz, 2013b). No Quadro 4, observa-se que os cargos comissionados do nível intermediário da gestão, DAS 1 e DAS 2, são ocupados em sua maioria por mulheres. Já os cargos referentes à alta direção da Fiocruz, presidente (DAS 6), vice-presidente, (DAS 5) e diretores (DAS 4), são ocupados por homens. 
Quadro 4 - Servidores/as com DAS, por unidade

\begin{tabular}{|c|c|c|c|c|c|c|c|c|c|c|c|c|c|}
\hline \multirow{2}{*}{ UNIDADE } & \multicolumn{2}{|c|}{ DAS 1} & \multicolumn{2}{|c|}{ DAS 2} & \multicolumn{2}{|c|}{ DAS 3} & \multicolumn{2}{|c|}{ DAS 4} & \multicolumn{2}{|c|}{ DAS 5} & \multicolumn{2}{|c|}{ DAS 6} & \multirow{2}{*}{$\begin{array}{l}\text { TOTAL } \\
\text { GERAL }\end{array}$} \\
\hline & $\mathbf{F}$ & $M$ & $\mathbf{F}$ & $M$ & $\mathbf{F}$ & $M$ & $\mathbf{F}$ & $M$ & $\mathbf{F}$ & $M$ & $\mathbf{F}$ & $M$ & \\
\hline IFF & 15 & 5 & 5 & 9 & 0 & 0 & 0 & 1 & 0 & 0 & 0 & 0 & 35 \\
\hline IPEC & 3 & 4 & 0 & 0 & 0 & 0 & 0 & 0 & 0 & 0 & 0 & 0 & 7 \\
\hline $\mathrm{COC}$ & 3 & 2 & 2 & 1 & 0 & 0 & 0 & 0 & 0 & 0 & 0 & 1 & 9 \\
\hline $\mathrm{ICICT}$ & 2 & 2 & 1 & 2 & 0 & 0 & 0 & 1 & 0 & 0 & 0 & 0 & 8 \\
\hline ENSP & 21 & 14 & 5 & 5 & 0 & 0 & 0 & 1 & 0 & 2 & 0 & 0 & 48 \\
\hline EPSJV & 2 & 1 & 2 & 2 & 0 & 0 & 0 & 0 & 0 & 0 & 0 & 0 & 7 \\
\hline PRESIDÉNCIA & 3 & 2 & 3 & 0 & 1 & 3 & 3 & 5 & 0 & 1 & 0 & 0 & 21 \\
\hline DIRAD & 5 & 3 & 2 & 1 & 0 & 0 & 1 & 0 & 0 & 0 & 0 & 0 & 12 \\
\hline DIPLAN & 0 & 0 & 0 & 0 & 2 & 1 & 1 & 1 & 0 & 0 & 0 & 0 & 5 \\
\hline DIRAC & 5 & 9 & 0 & 2 & 0 & 0 & 1 & 0 & 0 & 0 & 0 & 0 & 17 \\
\hline DIREH & 4 & 3 & 2 & 0 & 0 & 1 & 0 & 0 & 0 & 0 & 0 & 0 & 10 \\
\hline DIREB & 0 & 1 & 0 & 1 & 0 & 0 & 0 & 1 & 1 & 0 & 0 & 0 & 3 \\
\hline IOC & 37 & 20 & 9 & 4 & 0 & 0 & 1 & 0 & 0 & 0 & 0 & 0 & 72 \\
\hline CPgAM & 5 & 3 & 4 & 2 & 0 & 0 & 0 & 1 & 0 & 0 & 0 & 0 & 15 \\
\hline CPqGM & 7 & 6 & 0 & 1 & 0 & 0 & 0 & 0 & 0 & 0 & 0 & 0 & 14 \\
\hline CPqLMD & 0 & 0 & 0 & 0 & 0 & 0 & 0 & 0 & 0 & 0 & 0 & 0 & 0 \\
\hline CPqRR & 6 & 8 & 0 & 1 & 0 & 0 & 1 & 0 & 0 & 0 & 0 & 0 & 16 \\
\hline BIOMANGUINHOS & 11 & 7 & 2 & 3 & 0 & 0 & 0 & 1 & 0 & 0 & 0 & 0 & 24 \\
\hline FARMANGUINHOS & 4 & 7 & 4 & 2 & 0 & 0 & 0 & 1 & 0 & 0 & 0 & 0 & 18 \\
\hline INCQS & 12 & 12 & 6 & 4 & 0 & 0 & 0 & 1 & 0 & 0 & 0 & 0 & 35 \\
\hline CECAL & 1 & 2 & 0 & 0 & 0 & 0 & 1 & 0 & 0 & 0 & 0 & 0 & 4 \\
\hline TOTAL & 146 & 111 & 47 & 40 & 3 & 5 & 9 & 14 & 1 & 3 & 0 & 1 & 380 \\
\hline
\end{tabular}

Fonte: Direh/Fiocruz (2013).

No Quadro 5, verifica-se que a maioria das funções gratificadas (FG) é também ocupada por homens.

O Instituto Oswaldo Cruz (IOC) é a unidade com maior número de DAS, com 72 cargos comissionados, com 48 mulheres, sendo 1 com DAS 5, 1 com DAS 4, 37 com DAS 1 e 9 com DAS 2; e 24 homens, sendo 20 com DAS 1 e 4 com DAS 2, o que representa $18,95 \%$. A participação das mulheres nos cargos comissionados consolida uma gestão participativa e democrática da Fiocruz.

O Instituto Fernandes Figueira (IFF) e Instituto de Tecnologia em Imunobiológicos (Biomanguinhos) possuem o mesmo número de servidores/servidoras com FG, um total de 41, assim distribuídas: no IFF, 28 mulheres e 13 homens - o resultado aponta a permanência da feminização no setor hospitalar; e em Biomanguinhos, 21 homens e 20 mulheres - o resultado aponta a paridade na distribuição de função gratificada em uma unidade técnica de produção. 
cadernos pagu (46) Jeorgina G. Rodrigues e Maria Cristina S. Guimarães 215

Quadro 5 - Servidores/as com FG, por Unidade

\begin{tabular}{|l|c|c|c|c|c|c|c|}
\hline \multirow{2}{*}{ UNIDADE } & \multicolumn{2}{|c|}{ FG 1 } & \multicolumn{2}{c|}{ FG 2 } & \multicolumn{2}{c|}{ FG 3 } & \multirow{2}{*}{ TOTAL } \\
\cline { 2 - 7 } & F & M & F & M & F & M & GEL \\
\hline IFF & 0 & 0 & 9 & 4 & 19 & 9 & 41 \\
\hline IPEC & 0 & 0 & 3 & 2 & 7 & 3 & 15 \\
\hline COC & 1 & 1 & 0 & 2 & 3 & 2 & 9 \\
\hline ICICT & 1 & 4 & 2 & 2 & 4 & 6 & 19 \\
\hline ENSP & 3 & 1 & 8 & 6 & 6 & 12 & 36 \\
\hline EPSJV & 0 & 2 & 2 & 2 & 2 & 1 & 9 \\
\hline PRESIDENCIA & 3 & 2 & 7 & 7 & 1 & 3 & 23 \\
\hline DIRAD & 7 & 9 & 1 & 4 & 3 & 5 & 29 \\
\hline DIPLAN & 0 & 0 & 1 & 0 & 0 & 1 & 2 \\
\hline DIRAC & 0 & 15 & 0 & 10 & 3 & 10 & 38 \\
\hline DIREH & 3 & 3 & 1 & 1 & 0 & 1 & 9 \\
\hline DIREB & 0 & 1 & 0 & 1 & 0 & 0 & 2 \\
\hline IOC & 0 & 0 & 2 & 5 & 17 & 14 & 38 \\
\hline CPqAM & 1 & 0 & 4 & 1 & 3 & 6 & 15 \\
\hline CPqGM & 3 & 3 & 1 & 3 & 1 & 5 & 16 \\
\hline CPqLMD & 0 & 1 & 0 & 0 & 2 & 0 & 3 \\
\hline CPqRR & 0 & 0 & 3 & 3 & 0 & 1 & 7 \\
\hline BIOMANGUINHOS & 13 & 15 & 3 & 2 & 4 & 4 & 41 \\
\hline FARMANGUINHOS & 0 & 0 & 0 & 3 & 1 & 2 & 6 \\
\hline INCQS & 1 & 0 & 4 & 4 & 10 & 5 & 24 \\
\hline CECAL & 0 & 0 & 1 & 0 & 2 & 3 & 6 \\
\hline TOTAL & $\mathbf{3 6}$ & $\mathbf{5 7}$ & $\mathbf{5 2}$ & $\mathbf{6 2}$ & $\mathbf{8 8}$ & $\mathbf{9 3}$ & $\mathbf{3 8 8}$ \\
\hline
\end{tabular}

Fonte: Direh/Fiocruz (2013).

O Quadro 6 apresenta os cargos comissionados oficiais ocupados por servidores/as concursados/as com titulação de doutorado no momento do estudo. Na Fiocruz, além dos cargos comissionados oficiais existem aqueles que ocupam postos de tomada de decisão e não recebem o valor correspondente ao cargo de confiança. Em alguns casos, os servidores/as não recebem nenhum tipo de remuneração referente ao cargo ocupado.

Cabe ressaltar que atualmente na Fiocruz não existe equidade entre as unidades na distribuição de DAS, e, no caso do Instituto de Comunicação e Informação Científica e Tecnológica em Saúde (Icict), os servidores/servidoras que ocupam o cargo de vice-diretor não recebem DAS. Analisando o Quadro 6, evidenciase que os cargos de nível intermediário da gestão, DAS 1 e DAS 2, são ocupados em sua maioria por mulheres.

Apesar das evidências apontarem que as servidoras doutoras da Fiocruz têm participado cada vez mais das atividades 
216 A Fundação Oswaldo Cruz e a ciência no feminino

de C\&T nacional, elas ainda não avançaram nos cargos de alta direção da fundação, ou seja, é possível constatar o "teto de vidro" na instituição.

Quadro 6 - Servidore/as concursado/as com doutorado, com cargo comissionado

\begin{tabular}{|c|c|c|c|c|}
\hline $\begin{array}{c}\text { FUNÇĀO OU CARGO DE } \\
\text { CONFIANÇA }\end{array}$ & INSTÂNCIA & $\mathbf{F}$ & M & $\begin{array}{l}\text { TOTAL } \\
\text { GERAL }\end{array}$ \\
\hline \multirow[t]{2}{*}{ ASSISTENTE } & BIOMANGUINHOS & 1 & & 1 \\
\hline & $\mathrm{COC}$ & & 1 & 1 \\
\hline ASSISTENTE Total & & 1 & 1 & 2 \\
\hline \multirow[t]{3}{*}{ CHEFE } & BIOMANGUINHOS & & 1 & 1 \\
\hline & CPqGM & & 1 & 1 \\
\hline & IPEC & & 1 & 1 \\
\hline CHEFE Total & & & 3 & 3 \\
\hline \multirow[t]{5}{*}{ CHEFE DE DEPARTAMENTO } & CPqAM & 3 & 1 & 4 \\
\hline & ENSP & 1 & 1 & 2 \\
\hline & FARMANGUINHOS & 1 & & 1 \\
\hline & INCQS & 1 & & 1 \\
\hline & IOC & 1 & & 1 \\
\hline CHEFE DE DEPARTAMENTO Total & & 7 & 2 & 9 \\
\hline \multirow[t]{8}{*}{ CHEFE DE LABORATÓRIO } & BIOMANGUINHOS & 2 & & 2 \\
\hline & CPqGM & 3 & 2 & 5 \\
\hline & CPqRR & 2 & 1 & 3 \\
\hline & ENSP & 2 & & 2 \\
\hline & IOCT & 1 & & 1 \\
\hline & IOC & 15 & 12 & 27 \\
\hline & IPEC & 1 & 1 & 2 \\
\hline & PRESIDÊNCIA & & 1 & 1 \\
\hline CHEFE DE LABORATORIO Total & & 25 & 17 & 42 \\
\hline \multirow[t]{4}{*}{ CHEFE DE SERVICCO } & $\mathrm{COC}$ & 1 & & 1 \\
\hline & ENSP & 2 & 4 & 6 \\
\hline & IFF & & 1 & 1 \\
\hline & IPEC & 2 & & 2 \\
\hline CHEFE DE SERVICCO Total & & 5 & 5 & 10 \\
\hline \multirow[t]{2}{*}{ DIRETOR } & CPqLMD & & 1 & 1 \\
\hline & PRESIDÊNCIA & 1 & 1 & 2 \\
\hline DIRETOR Total & & 1 & 2 & 3 \\
\hline \multirow[t]{3}{*}{ FUNCCÃO GRATIFICADA } & IFF & 1 & & 1 \\
\hline & INCOS & 2 & 1 & 3 \\
\hline & IOC & 5 & 2 & 7 \\
\hline FUNÇÃO GRATIFICADA Total & & 8 & 3 & 11 \\
\hline \multirow[t]{11}{*}{ VICE-DIRETOR } & $\mathrm{COC}$ & & 2 & 2 \\
\hline & CPqAM & 1 & 1 & 2 \\
\hline & CPqGM & & 1 & 1 \\
\hline & CPqLMD & & 2 & 2 \\
\hline & CPqRR & & 2 & 2 \\
\hline & ENSP & 1 & 2 & 3 \\
\hline & EPSJV & & 1 & 1 \\
\hline & $\mathrm{ICICT}$ & 1 & 1 & 2 \\
\hline & INCQS & 1 & & 1 \\
\hline & $10 C$ & & 1 & 1 \\
\hline & IPEC & & 1 & 1 \\
\hline \multicolumn{2}{|l|}{ VICE-DIRETOR Total } & 4 & 14 & 18 \\
\hline \multicolumn{2}{|l|}{ TOTAL GERAL } & 52 & 47 & 99 \\
\hline
\end{tabular}

Fonte: Direh/Fiocruz (2013); Portal Transparência (2013). 


\section{Considerações finais}

A pesquisa realizada enfocou a participação feminina na pesquisa na Fiocruz, especialmente nos anos recentes. $\mathrm{O}$ universo de estudo foi delimitado pelos/as servidores/as da instituição com titulação de doutorado, independente de onde estejam locados, se em ensino e pesquisa ou em outras atividades de gestão. Optou-se em trabalhar com os servidores/as que entraram por concursos públicos realizados entre 1996 e 2010 e que no momento do estudo possuíam titulação de doutorado.

Utilizando-se a ferramenta ScriptLattes, foi realizada a extração de informações com base nos 570 currículos cadastrados na Plataforma Lattes, referente ao período 1996-2013. Essas informações foram migradas e tratadas em um software de mineração de dados, o VantagePoint ${ }^{\circledR}$, o que permitiu análises quantitativas da produção acadêmica e técnica feminina.

Este estudo tem como limitação principal a fonte de dados utilizada, o currículo Lattes que, a despeito de seu valor inconteste para esse tipo de análise de gênero, é de caráter declaratório e pode não representar fielmente a produção cientifica dos pesquisadores, além de ainda carecer de análise de consistência de dados. Antes de procurar pela completude e exaustividade, a pesquisa se dedicou ao delineamento de um cenário que, por certo, merece aprimoramentos e complementação. Quiçá possa também, a partir daqui, ser explorado por outros pesquisadores, de várias áreas do conhecimento.

\section{Referências bibliográficas}

ABreu, A. Pensando gênero e ciência nas Américas: a experiência da OEA. In: Encontro Nacional de Núcleos e Grupos de Pesquisas, Brasília, DF, 2006. Pensando gênero e ciência. Brasília, Secretaria Especial de Políticas para as Mulheres, 2006, pp.109-118.

Agrello, D. A.; GARG, R. Mulheres na física: poder e preconceito nos países em desenvolvimento. Revista brasileira de ensino de física, vol. 31, n¹, São Paulo, 2009, pp.1305-1310. 
AQUINO, E. M. L. Gênero e ciência no Brasil: contribuições para pensar a ação política na busca da equidade. In: Encontro Nacional De Núcleos E Grupos De Pesquisas, Brasilia, DF, 2006. Pensando gênero e ciência. Brasília, Secretaria Especial de Políticas para as Mulheres, 2006, pp.11-24.

ARAÚJO-JORGE, T. et al. Mulheres no IOC e na Ciência. 2013. [http://www.fiocruz.br/ioc/cgi/cgilua.exe/sys/start.htm?infoid =1732\&si $\underline{\mathrm{d}=32}$ - acesso em: 13 mar. 2013].

BRASIL. Ministério da Saúde. Secretaria de Atenção à Saúde. Departamento de Ações Programáticas Estratégicas. Política Nacional de Atenção Integral à Saúde da Mulher: princípios e diretrizes. Brasília, Editora do Ministério da Saúde, 2011.

BRASIL. Secretaria Especial de Políticas para as Mulheres. Plano Nacional de Políticas para as Mulheres. Brasília, 2005. [http://busms.saude.gov.br/bvs/publicacoes/pnpm_compacta.pdf acesso em: 23 abr. 2013].

Citeli, M. T. Mulheres nas ciências: mapeando campos de estudo. Cadernos Pagu (15), Campinas, Núcleo de Estudos de GêneroPagu/Unicamp, 2000, pp.39-75.

CNPQ. Mulheres bolsistas de produtividade em pesquisa conquistam direito.

[http://www.cnpq.br/web/guest/noticiasviews//journal content/56 INSTANCE_a6MO/10157/909274 - acesso em: 21 jun. 2013a].

CNPQ. Pioneiras da Ciência do Brasil. [<http://www.cnpq.br/web/guest/pioneiras-da-ciencia-do-brasil > acesso em: 21 jun. 2013b].

CostA, M. C. Ainda somos poucas: exclusão e invisibilidade na ciência. Cadernos Pagu (27), Campinas, Núcleo de Estudos de GêneroPagu/Unicamp, 2006, pp.455-459.

Etzkowitz, H.; KemelgoR, C.; Uzzi, B. Athena Unbound: The advancement of women in science and technology. Cambridge University Press, 2000.

EuROPEAN Commission. Benchmarking Policy Measures for Gender Equality in Science. Brussels, European Commission, 2008. 
FERREIRA, L. O. et al. Institucionalização das ciências, sistema de gênero e produção científica no Brasil (1939-1969). Hist. cienc. saúdeManguinhos vol.15, supl, Rio de Janeiro, Fiocruz, jun. 2008, pp.4371.

FONTENELE-MOURÃO, T. M. Mulheres no topo de carreira: flexibilidade e persistência. Brasilia, Secretaria Especial de Políticas para as Mulheres, 2006.

FÓRUM Econômico Mundial. Empoderamento das Mulheres: avaliação das disparidades globais de gênero. 2005. [<http://www.agende.org.br/docs/File/dados_pesquisas/cidadania/FE M\%20-

\%20avaliacao\%20das\%20disparidades\%20de\%20genero.pdf > acesso em 20 jun. 2013].

FundAÇão Oswaldo Cruz. Relatório de Gestão: 2011. Rio de Janeiro, Fiocruz, 2012.

. Relatório de Gestão: 2012. Rio de Janeiro: Fiocruz, 2013a.

. Diretoria de Recursos Humanos. Boletim de Recursos Humanos. Rio de Janeiro, 2013b. [http://www.direh.fiocruz.br/boletimrh/Boletim-de-RH-2013_222.pdf/ - acesso em: 20 set. 2013].

LANE, K. A.; GOH, J. X.; Driver-Linn, E. Implicit science stereotypes mediate the relationship between gender and academic participation. Sex Roles, vol. 66, New York, 2012, pp.220-234.

LEWIN, A. Y.; DUCHAN, L. Women in academia: a study of the hiring decision in departments of physical science. Science, New York, vol. 173, 1971, pp.892-895.

LETA, J. As mulheres na ciência brasileira: crescimento, contrastes e um perfil de sucesso. Estudos. avançados vol.17, n49, São Paulo, 2003, pp.271-284.

LETA, J.; LEWISON, G. The contribution of women in Brazilian science: a case study in astronomy, immunology and oceanography. Scientometrics vol.57, n³, Dordrecht, 2003, pp.339-53.

LiMA, B. S. O labirinto de cristal: as trajetórias das cientistas na Física. Revista Estudos Feministas vol. 21, n 3, Florianópolis, dez. 2013, pp.883-903. 
LOPES, M. M. As grandes ausentes das inovações em Ciência e Tecnologia. Cadernos Pagu (19), Campinas, Núcleo de Estudos de Gênero-Pagu/Unicamp, 2002, pp.315-318.

MASON, M. A.; GOULDEN, M. Marriage and baby blues: redefining gender equity in the academy. Annals of the American Academy of Political and Social Science, vol. 596, n 1,2004 , pp.86-103.

MEllo, M. L B C.; AMÂNCIO FILHO, A. A gestão de recursos humanos em uma instituição pública brasileira de ciência e tecnologia em saúde: o caso Fiocruz. Rev. Adm. Pública vol. 44, n 3, Rio de Janeiro, 2010.

Melo, H. P.; OliveirA, A. B. A produção científica brasileira no feminino. Cadernos Pagu (27), Campinas, Núcleo de Estudos de GêneroPagu/Unicamp, 2006, pp.301-331.

Moreira, M.; VelHo, L. Pós-graduação do Instituto Nacional de Pesquisas Espaciais numa perspectiva de gênero. Cadernos Pagu (35), Campinas, Núcleo de Estudos de Gênero-Pagu/Unicamp, 2010, pp.279-308.

Olinto, G. A inclusão das mulheres nas carreiras de ciência e tecnologia no Brasil. Inclusão Social vol. 5 n 1, Brasília, jul./dez. 2011, pp.6877.

OliveIRA, J. M.; AMÂNCIO, L. Liberdades condicionais: o conceito de papel sexual revisitado. Sociologia, Problemas e Práticas $n^{\circ} 40$, Oeiras, set. 2002.

OsADA, N. M.; CostA, M. C. A construção social de gênero na Biologia: preconceitos e obstáculos na biologia molecular. Cadernos Pagu, (27), Campinas, Núcleo de Estudos de Gênero-Pagu/Unicamp, pp.279-299.

PROGRAMA Pró-Equidade de Gênero. Oportunidades iguais respeito às diferenças. $\quad 2^{\mathrm{a} e d .} \quad$ Brasília, 2008. [http://www.bndes.gov.br/SiteBNDES/export/sites/default/bndes_pt/Ga lerias/Arquivos/programa proequidade.pdf - acesso em: 10 mar. 2013].

RECOMMENDATIONS from Civil Society on the Fundamental Role of Science, Technology, Engineering, Innovation, and Science Education within the Framework of Discussion for the Fourth Summit of the Americas, Set. 5, 2005, Buenos Aires, Argentina. 
[http://www.summit-americas.org/sirg_meet_2005.html - acesso em: 20 set. 2009].

Rodrigues, J. G. Gênero, Ciência \& Tecnologia e Saúde: apontamentos sobre a participação feminina na pesquisa na Fundação Oswaldo Cruz. Tese (Doutorado em Informação, Comunicação em Saúde) Fundação Oswaldo Cruz, Instituto de Informação Científica e Tecnológica em Saúde, Rio de Janeiro, 2014.

Roos, P. A.; GATTA, M. L. Gender (in)equity in the academy: subtle mechanisms and the production of inequality. Research in Social Stratification and Mobility vol.27, n³, Greenwich, CT, 2009, pp.177200.

Rossiter, M. The Matthew Matilda efect in science. Social Studies of Science vol. 23, n 2, London, 1993, pp.325-341.

SaAvedra, L.; TAVeirA, M. C.; Silva, A. D. A sub-representatividade das mulheres em áreas tipicamente masculinas: factores explicativos $e$ pistas para a intervenção. Rev. bras. orientac. prof. vol.11, $\mathrm{n}^{\circ} 1$, São Paulo, 2010, pp.49-59.

SCHIEBINGER, L. O feminismo mudou a ciência? Bauru, Edusc, 2001.

. Mais mulheres na ciência: questões de conhecimento. Hist. cienc. saúde - Manguinhos vol.15, supl, Rio de Janeiro, Fiocruz, 2008, pp.269-281.

ScotT, J. Gênero: uma categoria útil de análise histórica. Educação \& Realidade vol. 16, n 2. Porto Alegre, jul./dez. 1990, pp.71-99.

SHERIDAN, B. Strangers in a Strange Land: a literature review of women in Science. Boston, MA., Simmons Institute for Leadership and Change, Simmons College, 1998.

SMITH-DOERR, L. Women's work: Gender equality vs. hierarchy in the Life Sciences. Boulder, Lynne Rienner Publishers, 2004.

SummerS, L. H. Remarks at NBER Conference on Diversifying the Science \& Engineering Workforce. Cambridge, MA, 14 Jan. 2005.

TABAK, F. Apesar dos avanços: obstáculos ainda persistem. Cadernos de gênero e tecnologia vol.3, n¹0, Curitiba, 2007, pp.9-20. 
222 A Fundação Oswaldo Cruz e a ciência no feminino

UNESCO. A ciência para o século XXI: uma nova visão e uma base de ação. Budapeste e Santo Domingo. $3^{\text {a } e d . ~ B r a s i ́ l i a, ~ E d i c ̧ o ̃ e s ~}$ Unesco/ABIPTI, 2005.

VAZ, D. V. O teto de vidro nas organizações públicas: evidências para o Brasil. Economia e Sociedade vol. 22, $n^{\circ} 3$, Campinas, Instituto de Economia/Unicamp, dez. 2013, pp. 765-790.

VAZ, D. V. Segregação hierárquica de gênero no setor público brasileiro. Bol. mercado trabalho: conjuntura e análise $n^{\circ}$ 42, Brasília, fev. 2010, pp.27-36.

VelHo, L. A ciência e o seu público. Transinformação vol.9, n³, Campinas, set./dez. 1997, pp.15-32.

VelHo, L.; PROCHAZKA, M. No que o mundo da ciência difere dos outros mundos? Com ciência - Mulheres na Ciência no 50, dez. 2003. [http://www.comciencia.br/reportagens/mulheres/09.shtml - acesso em: 22 fev. 2011].

VelHo, L.; LEÓN, E. A construção social da produção científica por mulheres. Cadernos Pagu (10), Campinas, Núcleo de Estudos de Gênero-Pagu/Unicamp, 1998, pp.309-44.

XIE, Y.; Shauman, K. A. Women in Science: Career Processes and Outcomes. Cambridge, MA, Harvard University Press, 2004.

XU, Y. J. Gender disparity in STEM disciplines: a study of faculty attrition and turnover intentions. Res. High. Educ. New York, vol. 49, $\mathrm{n}^{\circ} 7$, 2008, pp.607-624. 